\title{
An Observational Case for the Prevalence of Roll Vortices in the Hurricane Boundary Layer*
}

\author{
IAn Morrison and Steven Businger \\ University of Hawaii at Manoa, Honolulu, Hawaii \\ Frank Marks and Peter Dodge \\ Hurricane Research Division, NOAA/AOML, Miami, Florida \\ Joost A. Businger \\ University of Washington, Seattle, Washington
}

(Manuscript received 5 April 2004, in final form 12 December 2004)

\begin{abstract}
Doppler velocity data from Weather Surveillance Radar-1988 Doppler (WSR-88D) radars during four hurricane landfalls are analyzed to investigate the presence of organized vortices in the hurricane boundary layer (HBL). The wavelength, depth, magnitude, and track of velocity anomalies were compiled through analysis of Doppler velocity data. The analysis reveals alternating bands of enhanced and reduced azimuthal winds closely aligned with the mean wind direction. Resulting statistics provide compelling evidence for the presence of organized secondary circulations or boundary layer rolls across significant areas during four hurricane landfalls. The results confirm previous observations of the presence of rolls in the HBL. A potential limitation of the study presented here is the resolution of the WSR-88D data. In particular, analysis of higher-resolution data (e.g., from the Doppler on Wheels) is needed to confirm that data aliasing has not unduly impacted the statistics reported here. Momentum fluxes associated with the secondary circulations are estimated using the covariance between the horizontal and vertical components of the wind fluctuations in rolls, with resulting fluxes 2-3 times greater than estimated by parameterizations in numerical weather prediction models. The observational analysis presented here, showing a prevalence of roll vortices in the HBL, has significant implications for the vertical transport of energy in hurricanes, for the character of wind damage, and for improvements in numerical simulations of hurricanes.
\end{abstract}

\section{Introduction}

Boundary layer roll vortices are known to be a common feature of the convective atmospheric boundary layer (ABL) and have been extensively studied observationally, analytically, and numerically (Kuettner 1971; LeMone 1973; Etling and Brown 1993; Mourad and Walter 1996; Weckwerth et al. 1997). High-

* School of Ocean and Earth Science and Technology Contribution Number 6601.

Corresponding author address: Steven Businger, Department of Meteorology, University of Hawaii at Manoa, 2525 Correa Rd., Honolulu, HI 96822.

E-mail: businger@hawaii.edu resolution observations provided by radar (Wurman and Winslow 1998, hereafter WW98) and synthetic aperture radar (SAR; Katsaros et al. 2000) supply evidence of similar circulations in the boundary layers of tropical cyclones (Fig. 1). The fine linear features seen across much of the SAR image in Fig. 1 have a wavelength of 2-4 km and are observed between convective bands in Typhoon Fengshen. Although the origin of the features observed by SAR has not been confirmed, it is reasonable to assume that they are the product of variations in surface wind speed and roughness associated with the vertical transport of momentum by boundary layer roll vortices. Damage swaths extending several hundred meters in the direction of the mean wind have been observed in the wake of hurricanes and midlatitude cyclones and attributed to wind gusts of extended duration (20 s) (Wakimoto and Black 1994; Mass et al. 


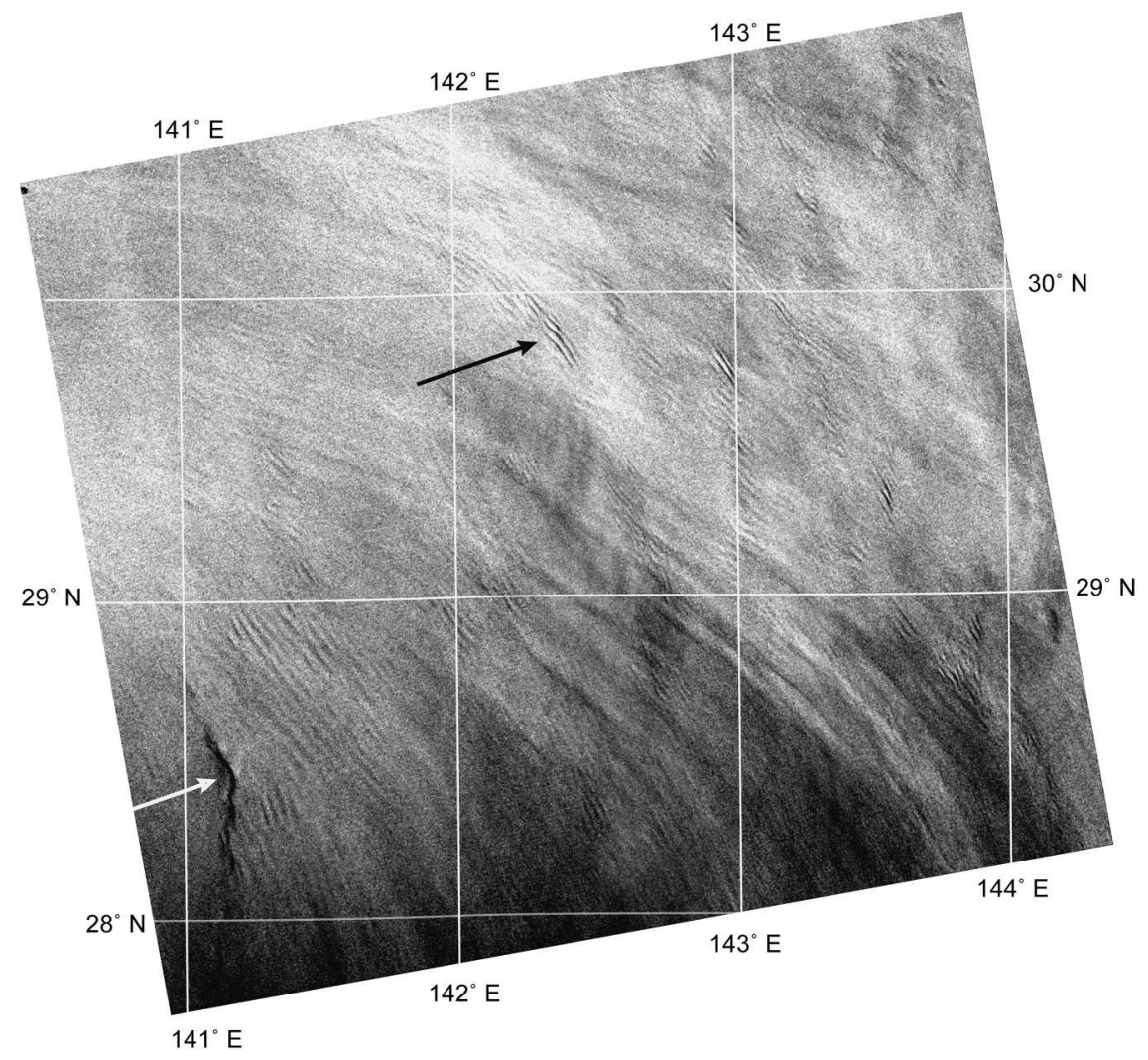

FIG. 1. RADARSAT-1 synthetic aperture radar (ScanSAR Wide B) image of Typhoon Fengshen showing evidence of finescale roll circulations across much of the image (e.g., black arrow). The image was taken at 0832:28 UTC 24 Jul 2002 (copyright Canadian Space Agency). The center of the typhoon is just to the southwest of the image at $\sim 28.3^{\circ} \mathrm{N}, 140.7^{\circ} \mathrm{E}$, with the signature of eyewall convection visible in the lower left of the image (white arrow). The image pixel resolution is $\sim 150 \mathrm{~m}$.

1995). It has been suggested that these damage patterns are consistent with downward momentum transport by helical roll circulations in the ABL (Fujita 1992; WW98).

In this paper we document the occurrence and the structural characteristics of organized roll vortices in the boundary layer of hurricanes through analysis of Weather Surveillance Radar-1988 Doppler (WSR-88D) data in four hurricane landfalls. For the purpose of this study the hurricane boundary layer (HBL) is defined as the region of the lower atmosphere that is directly affected by friction against the ocean surface and the transport of sensible and latent heat across the ocean surface. The extent of the hurricane boundary layer has been variously estimated as the depth of the inflow layer (Wroe and Barnes 2003) or the height of the inflection in the radial component of the wind profile (Brown 1980). If the extent of turbulent mixing in the HBL is taken as the top of the roll circulations, then a practical estimate for the extent of the HBL can be determined through the method of radar data analysis described in section 2 of this paper. The principal goal of this work is to improve our understanding of the vertical transport of energy across the HBL, which should lead to improvements in our ability to simulate intensity changes in these destructive storms, an ability that is remarkably lacking in current numerical weather prediction (NWP) models (Emanuel 1999).

Secondary circulations in the ABL have been previously documented through Doppler radar analysis. Kelly (1982), using data from a single-Doppler radar, found an alternating pattern of diffluence and confluence in the Doppler velocity fields associated with ABL rolls. Christian and Wakimoto (1989) related ABL rolls to cloud street location in light wind conditions in a lake-effect snowstorm using single-Doppler radar data. They found that ABL roll convergence areas produced radar echoes aligned with the cloud streets. Weckwerth et al. (1997) developed an objective method for determining roll characteristics in radar data and related wavelength and aspect ratio of observed rolls to convective instability and ABL depth. WW98 analyzed 
Doppler on Wheels (DOW) data gathered during Hurricane Fran (1996). They showed that the retrieved wind field in the lowest $200 \mathrm{~m}$ included features that resemble HBL rolls aligned along the mean wind vector. These features were small, narrow regions of enhanced flow flanked by large horizontal gradients $\left(10^{-1} \mathrm{~s}^{-1}\right)$.

Glendening (1996) utilized a large eddy simulation (LES) model to simulate ABL rolls under conditions of strong shear and low buoyancy conditions. The LES produced ABL rolls that transported significant amounts of momentum, moisture, and heat vertically. Brown and Zeng (2001) compared a two-layer similarity HBL model to GPS dropsonde observations in a hurricane environment. Their HBL model includes a parameterization of the HBL roll effects on wind profile, momentum, and heat fluxes. Their results show that the HBL model matches the GPS dropsonde observations well, and this favorable comparison is due to the inclusion of the nonlinear HBL roll effects. Currently, most numerical weather prediction (NWP) models do not employ ABL roll effects in the transport of momentum, heat, and moisture in the ABL. Instead, they use an eddy-viscosity model that parameterizes the turbulent transport homogeneously throughout the ABL. The first attempt to provide an analytical description of roll vortices in hurricanes was undertaken by Foster (2005), in collaboration with this observationally based paper. Foster concludes that the HBL is ideally suited for roll formation because of an instability associated with an inflection point that is characteristically present in the radial component of HBL wind profiles. His predictions regarding the characteristics of the rolls agree with the observational results presented in this paper.

\section{Methods}

The WSR-88D radar completes a volume scan in 6 min when scanning in precipitation mode. The volume scan is composed of 14 conical scans at elevation angles ranging from $0.5^{\circ}$ to $19.5^{\circ}$ over a domain within $125 \mathrm{~km}$ of the radar with a radial resolution of $250 \mathrm{~m}$. Crum and Alberty (1993) provide additional technical and performance specification for the WSR-88D radar. A Fourier analysis of the velocity-azimuth display (VAD) is used to estimate profiles of mean wind speed and direction for each elevation and radius in a volume scan (Browning and Wexler 1968). At a given scan elevation and radius, mean radial velocity (first harmonic) is a sinusoidal function of azimuth angle, with the amplitude and phase of the sine curve providing the mean wind speed and direction (Fig. 2).

To maximize the vertical resolution and minimize

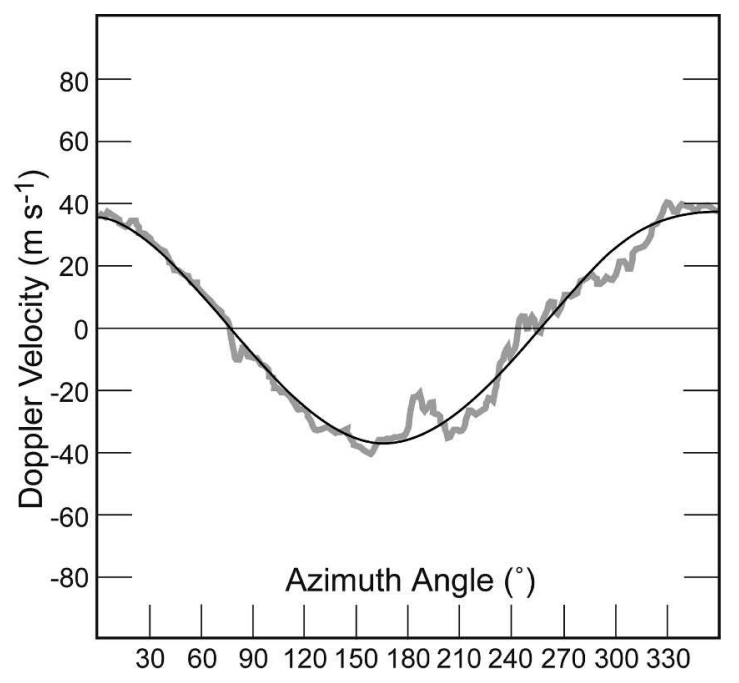

FIG. 2. The gray line is the radial velocity at $5-\mathrm{km}$ distance from the radar. The black line is the sinusoidal component of the radial velocity (first harmonic). Data shown are from the Puerto Rico WSR-88D at 2203 UTC 21 Sep 1998, taken in Hurricane Georges.

ground clutter contamination, horizontal wind speed and direction estimates were computed for the 20 radial bands at 250-m resolution between 5.0 and $9.75 \mathrm{~km}$ at each elevation angle (Marks et al. 1999). This approach yields horizontal wind estimates starting $62 \mathrm{~m}$ above the radar (so typically $100 \mathrm{~m}$ above the ground) and extending to $3.4 \mathrm{~km}$ with estimates every $\sim 25 \mathrm{~m}$ on average. The angular beamwidth of the WSR-88D is $\sim 0.95^{\circ}$; therefore, at a range of $9.8 \mathrm{~km}$ the beamwidth is $\sim 162 \mathrm{~m}$, which represents a limit to the horizontal and vertical resolution of the radar data at the maximum range used in this study. This resolution is comparable to the resolution of $160 \mathrm{~m}$ at $10 \mathrm{~km}$ reported by WW98, though their gate-to-gate range resolution of 75 $\mathrm{m}$ is significantly better than the range resolution of $\sim 200 \mathrm{~m}$ of the WSR-88D.

Velocity estimates in each radial band fluctuate about a mean profile with an rms of $2-3 \mathrm{~m} \mathrm{~s}^{-1}$. Smooth profiles were constructed using a Gaussian-weighted filter with an $e$-folding distance of $\sim 75 \mathrm{~m}$. Tangential $\left(V_{t}\right)$ and radial $\left(V_{r}\right)$ wind components were derived using the storm track and radar position (Fig. 3a). Here $V_{t}$ is the component of the wind oriented perpendicular, in a counterclockwise direction, to a radial line extending from the storm center determined from the track data, and $V_{r}$ is the component of the wind oriented parallel to a radial line extending from the storm center.

VAD wind profiles were compared to available radiosonde balloon profiles released by the National Weather Service in close proximity to the radar site. The tangential and radial wind profiles from the VAD method were in reasonable agreement with those of the 

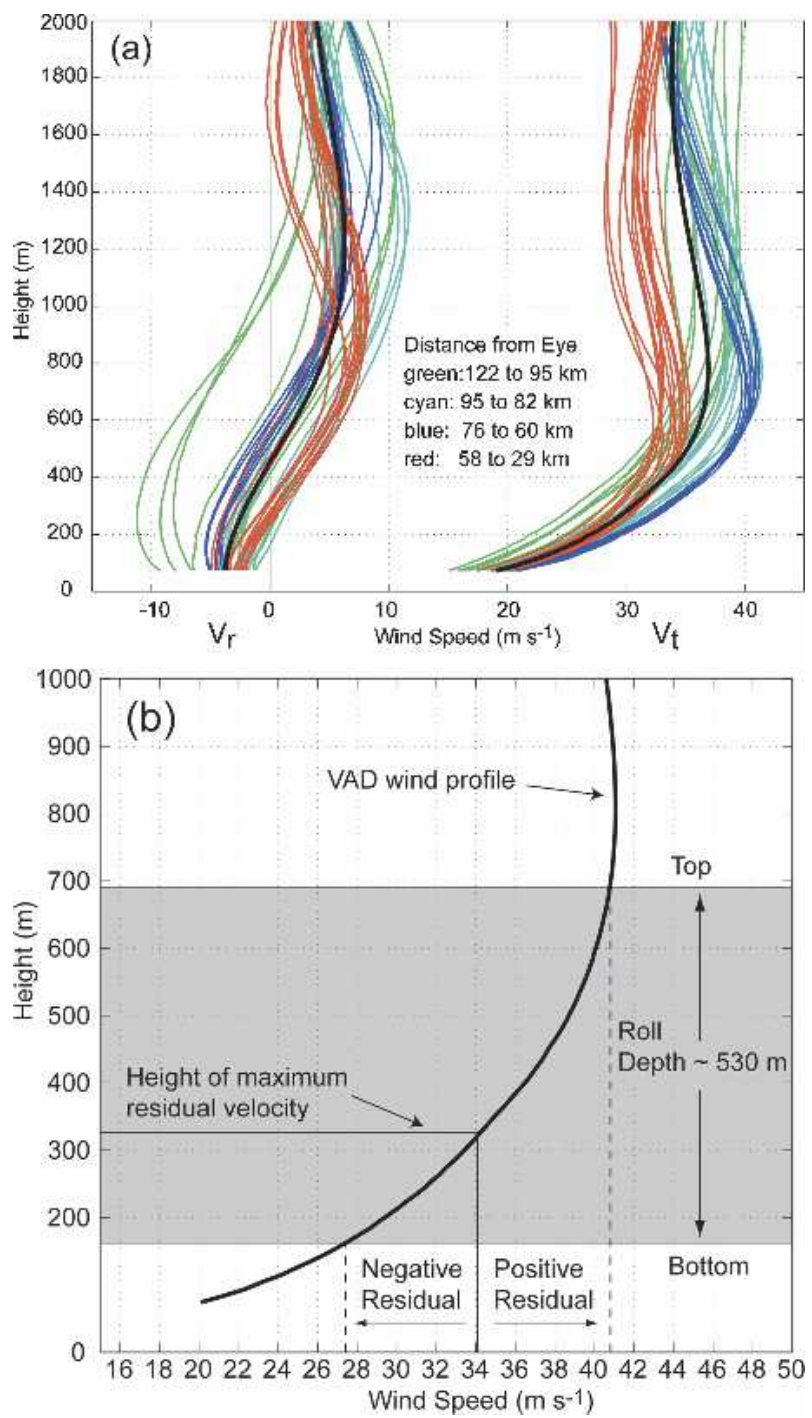

FIG. 3. (a) VAD tangential $\left(V_{t}\right)$ and radial $\left(V_{r}\right)$ wind profiles in Hurricane Bonnie at color-coded distances from 29 to $122 \mathrm{~km}$ from the eye. The heavy black line is the mean of the component VAD wind profiles taken in Bonnie. (b) Illustration of the method used to estimate the HBL roll depth from the VAD profile and the characteristics of the HBL roll. The data shown are for a HBL roll observed in Hurricane Bonnie at 0534 UTC 27 Aug 1998.

radiosonde (rms difference $\sim 1$ and $3 \mathrm{~m} \mathrm{~s}^{-1}$, respectively). Slightly larger differences in the radial component can be explained in part by noting that the radial velocity varies substantially with radial distance to the eye. As an areal average, the VAD-derived radial velocity is sensitive to the proximity of the hurricane eye.

To isolate velocity anomalies associated with organized HBL rolls from the mean background flow, the Doppler radial velocity is subtracted from the first harmonic of the VAD-derived velocity in each radial band to produce a residual velocity field for each elevation angle scan from $0.5^{\circ}$ to $5.5^{\circ}$ (Fig. 2). The lowest six scans are used to ensure the data being processed are within the boundary layer and not the free atmosphere. A threshold of $\pm 3 \mathrm{~m} \mathrm{~s}^{-1}$ is applied to the residual velocity field to emphasize the structure in the field (Fig. 4 ). Doppler radars can only observe the component of the wind along radar beam. Therefore, the velocity residuals tend to be most pronounced within $15^{\circ}$ of the mean HBL wind direction, with alternating bands of enhanced and reduced azimuthal velocities visible (Figs. 2 and 4). Pairs of adjacent positive and negative velocities were noted on each residual velocity scan, with the scans slicing at a low elevation angle through the features. When such pairs were observed in successive scans, their signatures were analyzed to accumulate evidence of coherent structures, or roll vortices, in the HBL (Fig. 4). Observations of wavelength are made when the radar beam is directed parallel to the long axis of the rolls; therefore it is suggested that the relevant limitation on resolution is that of radar beamwidth at maximum range $(\sim 160 \mathrm{~m})$, rather than the range gate spacing. It is acknowledged that features with a wavelength less that $\sim 320 \mathrm{~m}$ are not resolved in the WSR$88 \mathrm{D}$ radar data at the maximum range of $10 \mathrm{~km}$, and the amplitudes of features with wavelengths in the 400-600 $\mathrm{m}$ range are not fully resolved (Carbone et al. 1985).

HBL roll motion is estimated using the center of the anomaly pairs for successive scans. The low elevation angle of radar beam tilts and short range $(<10 \mathrm{~km})$ ensure the sampling stays within the HBL and also prevent the beam tilt from significantly affecting the HBL motion calculation. To exclude the possibility that some of the motion could be attributed to tilting of the phenomena in the vertical, comparisons were made of estimated motion for HBL rolls moving toward the radar and away from the radar and these showed no significant difference in speed.

The depth of the roll circulations can be estimated with reference to the VAD wind profiles, assuming vertical transport associated with the rolls is responsible for the residual velocity signatures. The average height of velocity residual pairs taken from successive residual velocity scans was plotted onto the VAD wind profile for the same time period (Fig. 3b). The levels above and below this height, where the mean wind profile matches the positive and negative wind residuals, provide an estimate of the depth of the roll circulation (shading in Fig. 3b).

\section{Results}

Three storms are examined, Fran (1996), Bonnie (1998), and Georges (1998), using WSR-88D data from Wilmington, North Carolina; Morehead City, North 


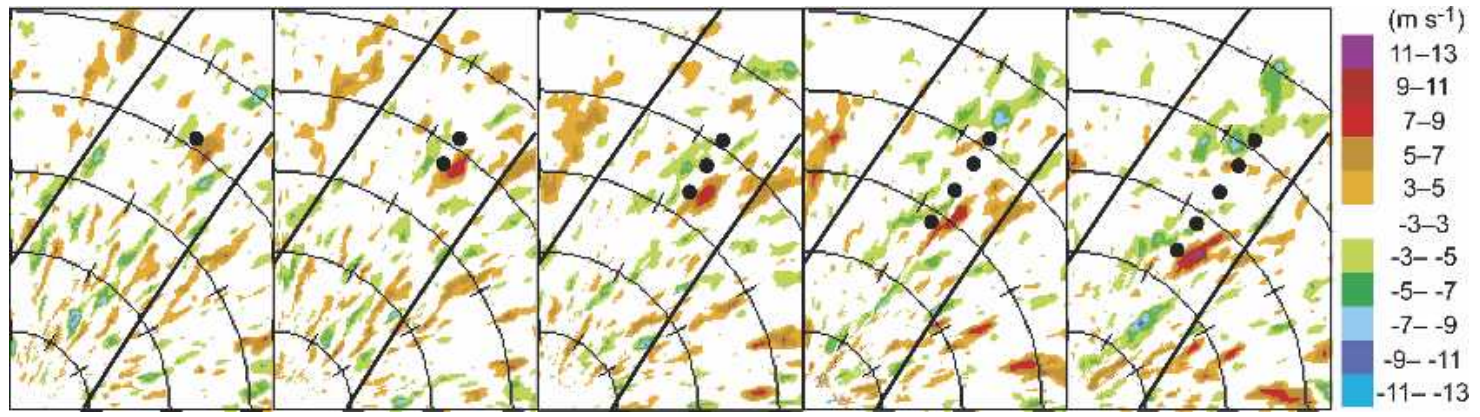

FIG. 4. Track of residual velocity pair (dots) in a sequence of radar scans taken every $25 \mathrm{~s}$. Thin black rings show constant radial distance from the radar (every $2 \mathrm{~km}$ ) and heavy black lines indicate approximate orientation of local isobars. The eye of Hurricane Fran is $87 \mathrm{~km}$ south-southeast of the radar location at the time of the first scan, 2233 UTC 5 Sep 1996.

Carolina; Puerto Rico; and Key West, Florida. Georges' path crossed Puerto Rico and Key West. Radar data were analyzed from the time of the first identified HBL roll circulation through hurricane landfall. Following the methodology described in section 2, 149 HBL rolls were documented during the four hurricane landfalls with 56, 44, 24, and $25 \mathrm{HBL}$ rolls identified in Fran, Bonnie, Georges (Key West), and Georges (Puerto Rico), respectively (Fig. 5).

Fran was a category 3 hurricane on the SaffirSimpson intensity scale while it was approaching North Carolina and dropped to category 2 near landfall. Fran passed within $28 \mathrm{~km}$ of Wilmington and the radius of maximum winds crossed the radar (Fig. 5). The first HBL rolls were identified $105 \mathrm{~km}$ from the eye.

Bonnie was a category 2 hurricane during the sampling period and passed within $30 \mathrm{~km}$ of Morehead City. The radar remained outside the radius of maximum winds for Bonnie. The first HBL rolls were identified in Bonnie $<120 \mathrm{~km}$ from Morehead City.

Georges passed directly over Puerto Rico as a category 3 storm and $25 \mathrm{~km}$ from Key West as a category 2 storm. HBL rolls were identified out to distances of 135 and $80 \mathrm{~km}$ from Puerto Rico and Key West, respectively. After the first roll was identified in each of the four hurricane landfalls, a large fraction of the subsequent scans (35\% to $69 \%$ ) showed evidence of roll vortices (Fig. 5). Evidence for rolls (adjacent pairs of positive and negative residual velocities) consistently vanished in the radar scans whenever convective rainfall crossed the radar scan. This observation is in conflict with WW98 who report roll signatures in the eyewall of Hurricane Fran at landfall over North Carolina; discussion of these differences is deferred to section 4 .

Histograms for wavelength, depth, aspect ratio, and the positive residual velocity (Fig. 6) each display a lognormal distribution, with mean values of $\sim 1450 \mathrm{~m}$, $660 \mathrm{~m}, 2.4$, and $7 \mathrm{~m} \mathrm{~s}^{-1}$, respectively. The geometric standard deviation, $s$, for the lognormal distribution fit to the data usually ranges from 0.2 to 1.0 , which suggests that the presented data are lognormal. The HBL rolls create adjacent positive and negative residual velocities that result in substantial vertical vorticity $\left(\sim 0.011 \mathrm{~s}^{-1}\right.$ over horizontal scales of $\left.1.5 \mathrm{~km}\right)$. The positive residual velocities, associated with downward leg of the transverse roll circulation, are shown in Fig. 6c because they have been implicated as the source of damage swaths in the wake of hurricanes (WW98).

The aspect ratio, defined as the wavelength divided by depth, ranges from 1 to 4.5 for all cases with a mean of 2.4. WW98 reported aspect ratios averaging 1.7 for HBL rolls identified in Hurricane Fran (1996), and Etling and Brown (1993) reported aspect ratios ranging from 2 to 15 in their literature review. The aspect ratio increases with increasing shear in the BL. Analytical results show that the aspect ratio is sensitive to the mean shear profile as well as the height of the inflection point (Foster 2005). In addition, the aspect ratio data show a general increase in variability with distance away from the eyewall.

The inflection point in the radial wind $\left(V_{r}\right)$ profiles, where the curvature goes from positive to negative, provides a depth estimate of the HBL (Fig. 3a) (Brown 1980). For Hurricane Bonnie the HBL depth varies from $\sim 800$ to $1200 \mathrm{~m}$, whereas estimates of the depth of the HBL roll circulations average $660 \mathrm{~m}$. The depth of the hurricane inflow layer can be determined by examining profiles of the mean radial wind component $\left(V_{r}\right)$. For Hurricane Bonnie the depth of the inflow layer ranges from $\sim 200$ to $\sim 1100 \mathrm{~m}$, with a mean inflow depth of $\sim 450 \mathrm{~m}$. These data suggest that the depth of the rolls is slightly greater than the inflow layer depth and slightly less than the depth of the HBL estimated from the height of the inflection point in the radial wind profiles. The inflow layer depth and height of the inflection point increase with distance away from the eye 

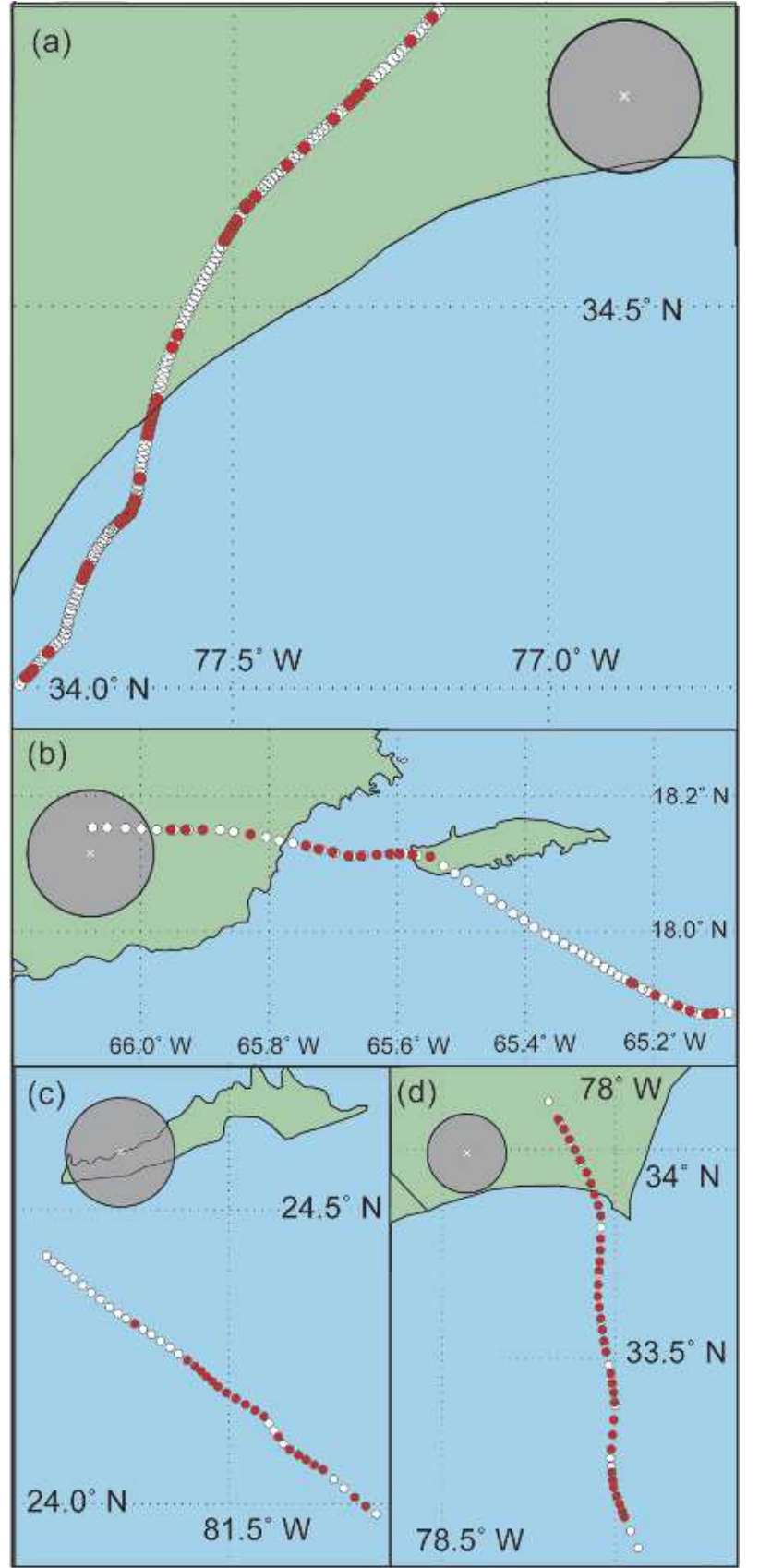

FIG. 5. Maps showing hurricane tracks for (a) Bonnie, (b) Georges (Puerto Rico), (c) Georges (Key West), and (d) Fran, and radar scan areas (large gray circles with radius $\sim 10 \mathrm{~km}$ ). Red track circles indicate the presence of roll vortices within the gray area of the radar scans at the time when the center of the hurricane was located at the track circle. White track circles indicate no roll vortices were observed in the associated radar scans.

(Fig. 3), as do depth and aspect ratio of the rolls (not shown).

Analysis of HBL roll motion suggests that the tracks curve with the gradient wind, moving roughly parallel to inferred isobars and the mean wind (Fig. 4). When the HBL roll motion is compared to that of the mean HBL wind direction, it is found that on average the HBL rolls are oriented $\sim 4^{\circ}$ to the left of the mean wind (Fig. 6e), in agreement with theory (Foster 2005). In contrast to the other histograms shown in Fig. 6, the fit of the HBL wind direction minus roll orientations is Gaussian rather than lognormal. The implication is that orientation of the rolls relative to the mean wind direction in the layer is normally distributed.

\section{Estimation of roll momentum flux}

VAD profiles are used in conjunction with the observed characteristics of HBL rolls to estimate the momentum flux associated with the HBL roll circulations. The momentum flux $\left(F_{m}\right)$ is equal to the covariance between the horizontal $(u)$ and vertical $(w)$ velocity fluctuations, $F_{m}=\overline{u w}$. Averaging can be performed in time or in space to obtain the mean and fluctuating components of the wind (Fleagle and Businger 1980). In this analysis the VAD provides an areal average, and the residual velocity fluctuations produced by the rolls can be taken as the fluctuating component $(u)$ of the wind in the direction of the mean wind (e.g., Fig. 2).

The vertical velocity fluctuations $(w)$ associated with the HBL rolls are estimated using two approaches. First a mixing-length approach is taken (Stull 1988) in which the variance of $w, \sigma_{w}=k z^{\prime}(d U / d z)$, where $k$ is the von Kármán constant $(0.4), d U / d z$ is the magnitude of the shear over the depth of the roll, and $z^{\prime}$ is the depth of the HBL roll circulation (e.g., Fig. 3b). Using mean values for the roll circulation (Fig. 6), the mean vertical velocity fluctuation, $\sigma_{w}=\left(\overline{w^{2}}\right)^{1 / 2}$, is $\sim 3 \mathrm{~m} \mathrm{~s}^{-1}$.

A second approach for estimating the vertical velocity fluctuations is to approximate the HBL rolls as twodimensional circulations (Lemone 1973). Continuity can then be applied to derive an estimate of the vertical velocity: $(d v / d y+d w / d z)=0$, assuming uniformity in the $x$ direction, the direction of the mean flow, and that the magnitude of the convergence in $v$ is proportional to the convergence seen in the radial component of the radar data. Again, taking typical observations for the rolls (Fig. 6), the mean $\sigma_{w}$ is $\sim 3 \mathrm{~m} \mathrm{~s}^{-1}$. This estimate is consistent with the result using the mixing length approach above. A comparison of results of the two methods for each roll observation shows reasonable agreement between these approaches, lending credence to the suggestion that an estimate of the momentum flux associated with the roll circulations can be obtained from the radar observations.

The momentum flux associated with each roll circulation $(\overline{u w})$ is calculated using the estimate for $u$ from the residual velocity field and $w$ from the mixing-length approach. An areal average of the momentum flux 

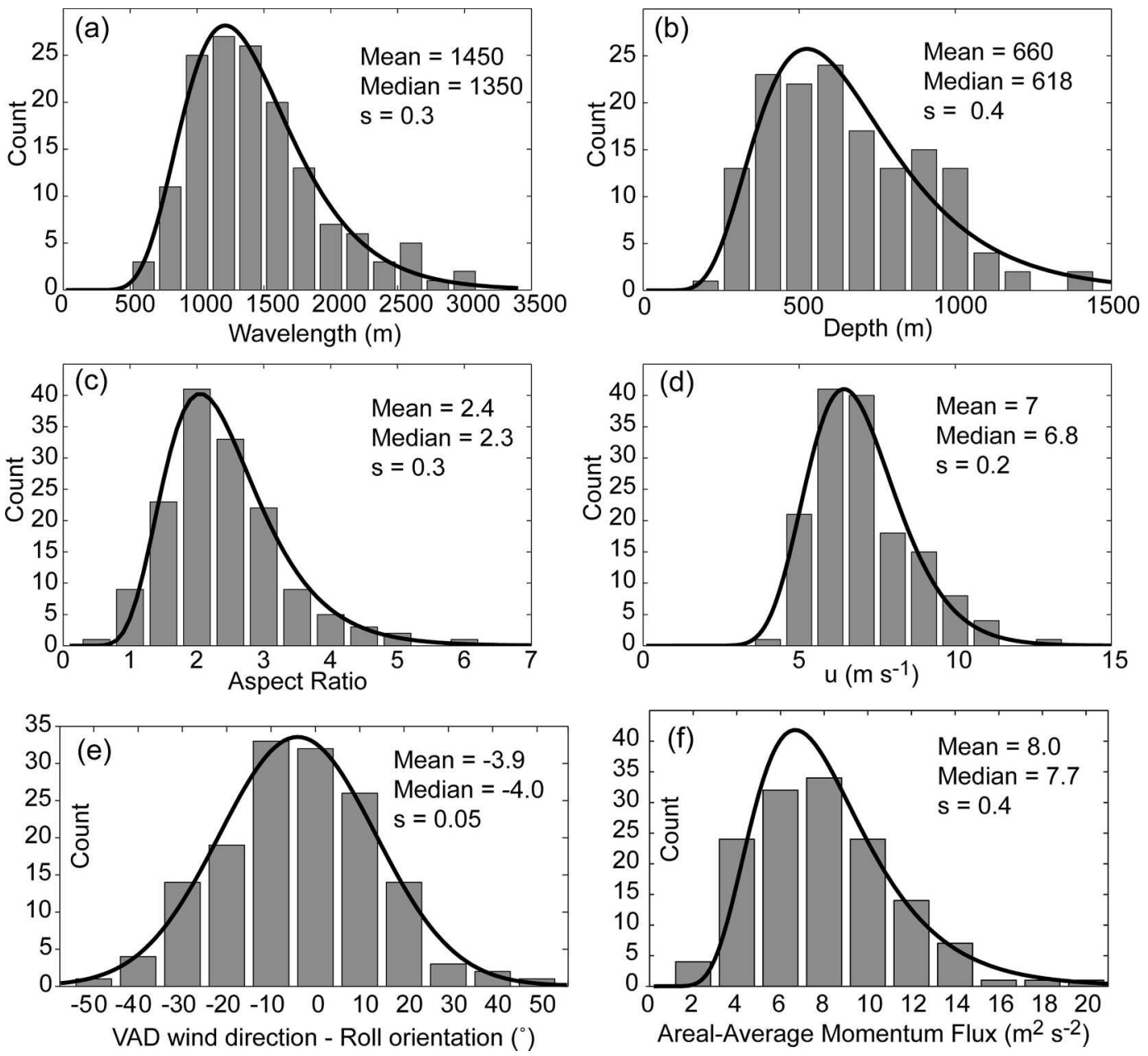

FIG. 6. Histograms of (a) wavelength, (b) depth, (c) aspect ratio, (d) positive residual velocity $u$, (e) orientation of the HBL rolls vs the mean HBL wind direction, and (f) momentum flux averaged over the roll. Curve fit is shown; s: geometric standard deviation.

across the HBL rolls can be obtained by assuming that the momentum flux varies with the vertical velocity component across the roll and thus is roughly sinusoidal, with maxima in the updraft and downdraft portions of the roll and tending to zero in between. The resulting areal-average momentum flux is $\sim 8 \mathrm{~m}^{2} \mathrm{~s}^{-2}$ for all of the rolls analyzed. Again the areal-mean flux exhibits a nearly lognormal distribution (Fig. 6f).

The areal-average momentum flux determined from the rolls is an appropriate estimate to compare with fluxes predicted by current NWP model parameterizations. To do this we need radar measurements over the sea. Air parcels in Fran had a long trajectory over land in approach to the radar, while air parcels over Puerto Rico in Georges experienced the impact of complex terrain. Therefore, data from Fran and Georges in Puerto Rico were eliminated to reduce the influence of the variability of friction and terrain on the momentum- flux results. The areal-average momentum flux in this reduced dataset varies from $\sim 5$ to $25 \mathrm{~m}^{2} \mathrm{~s}^{-2}$ (Fig. 7). For comparison, an estimate of the momentum flux, based on HBL parameterizations used in current NWP models of homogeneous turbulent diffusion, was calculated using 100-m VAD winds, assuming neutral conditions and a logarithmic profile in the surface layer. The results show the average momentum flux associated with the HBL rolls is $2-3$ times greater than estimates from model parameterization of homogeneous turbulent diffusion (Fig. 7).

Estimates of roll-induced momentum fluxes, prior to application of areal averaging, ranged from 7 to nearly $50 \mathrm{~m}^{2} \mathrm{~s}^{-2}$ and averaged $\sim 19 \mathrm{~m}^{2} \mathrm{~s}^{-2}$, significantly greater than the areal-average momentum flux shown in Fig. 7. These larger values may be appropriate to reference when considering the variability of the surface winds, their damage potential, and the impact of 


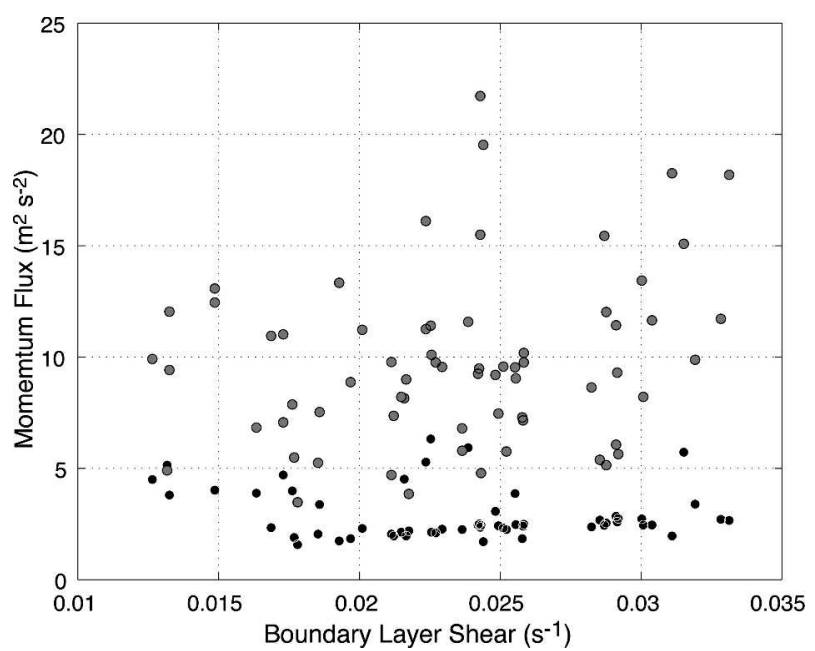

FIG. 7. Scatterplot of the areal-average momentum flux associated with the HBL rolls vs the HBL shear for Hurricanes Bonnie and Georges (Key West) (gray dots). For comparison, the black dots show the momentum flux calculated from 100-m wind from the VAD wind profiles alone, assuming neutral stability in the surface layer and a roughness length of $0.3 \mathrm{~m}$.

heating associated with the dissipation of turbulence kinetic energy at the air-sea surface (Bister and Emanuel 1998; Businger and Businger 2001).

\section{Conclusions and discussion}

\section{a. Summary of conclusions}

Doppler velocity data from WSR-88D radars during hurricane landfall are analyzed to investigate the pres- ence of organized eddies in the hurricane boundary layer (HBL). The analysis and resulting statistics provide compelling evidence for the presence of organized secondary circulations or boundary layer rolls in hurricanes. A large fraction of radar scans (35\% to 69\%) showed evidence of roll vortices, following identification of the first roll in each of the four hurricane landfalls. A schematic that summarizes the results of the analysis, with reference to the nonlinear theory, is presented in Fig. 8.

Histograms for wavelength, depth, aspect ratio, and residual velocity each display a lognormal distribution, with mean values of $\sim 1450 \mathrm{~m}, 660 \mathrm{~m}, 2.4$, and $7 \mathrm{~m} \mathrm{~s}^{-1}$, respectively. The mean gradient between adjacent positive and negative velocity residuals corresponds to a horizontal wind shear of $\sim 14 \mathrm{~m} \mathrm{~s}^{-1}$ over $\sim 725 \mathrm{~m}$ (wavelength $\sim 1450 \mathrm{~m}$ ) and a shear component of vertical vorticity of $1.1 \times 10^{-2} \mathrm{~s}^{-1}$. The mean momentum flux averaged over the rolls is $\sim 8 \mathrm{~m}^{2} \mathrm{~s}^{-2}$ for all the rolls analyzed and also exhibits a nearly lognormal distribution.

\section{b. Discussion}

J. Wurman (2004, personal communication) notes the appearance of linear features with wavelengths as short as $200 \mathrm{~m}$ in the lowest part of the HBL near the radar in DOW scans. Farther from the DOW radar (5-10-km range) the wavelength and depth of the dominant features increase. These observations are not inconsistent with those documented with the WSR-88D. For a feature to be included in the statistics compiled in

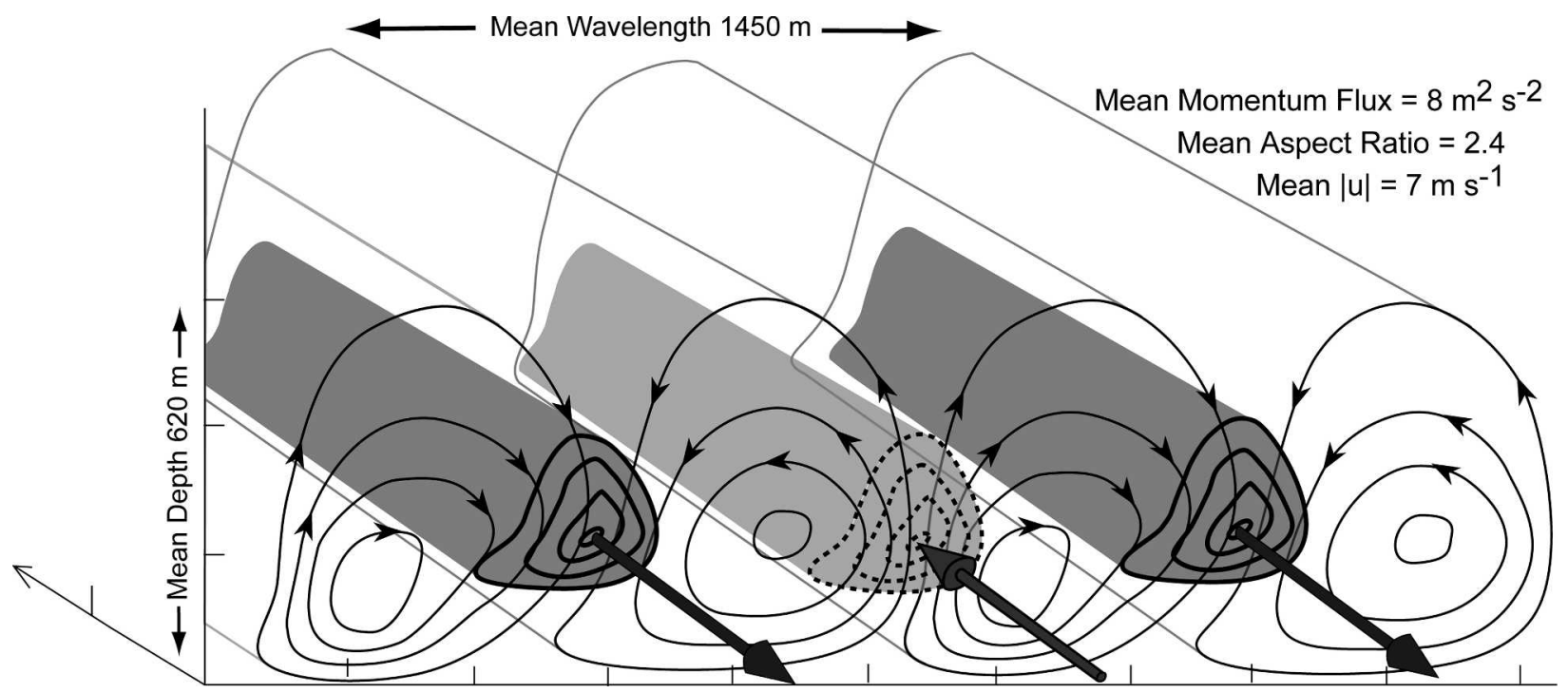

FIG. 8. Schematic depicting hurricane boundary layer rolls observed during four hurricane landfalls. Streamline arrows indicate transverse flow, with high (low) momentum air being transported downward (upward). Shaded arrows and bold contours indicate the positive (red) and negative (blue) residual velocities [R. Foster 2004, personal communication; after Brown (1974) and WW98]. 
the present study, it had to exhibit temporal continuity in consecutive scans. Smaller-scale features in the WSR-88D data were generally less traceable in subsequent scans. However, analysis of higher-resolution data is needed to confirm the potential impact of data aliasing on the statistics reported here.

The features documented in this research exhibit a linear structure nearly aligned with the mean wind in the HBL. Observations of wavelength are made when the radar beam is directed parallel to the long axis of the rolls; therefore it is suggested that the relevant limitation on resolution is that of radar beamwidth at maximum range $(\sim 160 \mathrm{~m})$. Features with a wavelength less that $\sim 320 \mathrm{~m}$ are not resolved in the WSR-88D data at the maximum of $10 \mathrm{~km}$, and the amplitudes of features with wavelengths in the 400-600-m range are not fully resolved (Carbone et al. 1985). Nearest the radar, where a narrower radar beam is observing lower in the HBL, the horizontal scale of the velocity anomalies appears to diminish. It was noted that smaller-scale, shallower features generally lack temporal coherence in the WSR-88D data. This finding may be sensitive to data resolution, and it is suggested that these smaller features deserve attention in future research. Recent improvements in the resolution of the DOW, through oversampling and a finer gate-to-gate range resolution ( 25 to $37 \mathrm{~m}$ ), make these data a promising choice to address the question of whether data aliasing to larger scales impacts the results of the research presented here.

Young et al. (2002) cite five mechanisms that can result in quasi-two-dimensional structures observed in the ABL: (i) shear-driven surface-layer turbulence, (ii) buoyancy-driven turbulence in a sheared surface layer, (iii) and (iv) two environmentally determined modes of boundary layer rolls with vertical scales comparable to the depth of the mixed layer, and (v) elongated features forced by gravity waves.

The characteristics of the observed HBL roll vortices (e.g., orientation relative to mean flow, occurrence under neutral or unstable conditions) cannot be explained by gravity waves. Results of an analytic model of coherent structures in surface layers under constant shear conditions show that hairpin vortices result as one moves away from the surface boundary (Bakas et al. 2001). Hairpin vortices, also know as streaks, undergo a continuous cycle of generation, strengthening, decay, and reformation, and their characteristic lifetimes (up to $20 \mathrm{~min}$ ) decrease with distance away from the surface (Bakas et al. 2001; Young et al. 2002). It is suggested that surface-layer shear mechanisms do not adequately explain the temporal and spatial characteristics associated with the HBL roll features observed in radar and
SAR data (Fig. 1). However, hairpin vortices may play an important organizing role by forcing roll instabilities that are near the most rapidly growing normal mode. The interaction between hairpin vortices and HBL rolls may help explain the observed variability and lognormal distribution seen in the wavelength, depth, and residual velocity statistics presented in this paper (Fig. 6).

The lognormal distribution evident in the results implies that the variable is exponentially dependent on another variable that is normally distributed and that the probable magnitude of any incremental change in this second variable is proportional to its instantaneous value. For example, the stress in the surface layer is nearly lognormally distributed (Powell et al. 2003) and the stress is related to the vertical shear of the horizontal wind, whose magnitude is a function of the height above the surface. The height of the inflection point, which determines the depth of the roll circulation, in turn is related to the distribution of wind shear in the HBL. Therefore, the distribution of the depth, wavelength, and residual velocities may be expected to display a lognormal character.

The HBL rolls in this study are identified in a highly sheared environment in which the HBL is capped by subsiding air between spiral bands of organized deep convection outside the eyewall. It is suggested that mechanical mixing as a result of the shear will tend to produce near neutral or slightly unstable thermodynamic conditions. The strong shear in the HBL under these circumstances is observed to result in an inflection point in the radial wind profile (Fig. 3a). Theoretical results suggest that this circumstance is conducive to the development of the HBL rolls (Lilly 1966; Brown 1980; Foster 2005).

Rolls with an average wavelength of $\sim 600 \mathrm{~m}$ and residual velocity of $\sim 15 \mathrm{~m} \mathrm{~s}^{-1}$ were documented by WW98 in the eyewall of Hurricane Fran. Although their average wavelength falls within the range observed in this study it is more than a factor of 2 smaller than the average (Fig. 6a). Since both radars (WSR$88 \mathrm{D}$ and DOW) have a similar beamwidth $(\sim 160 \mathrm{~m}$ at 10-km maximum range), data aliasing is not likely to fully account for the difference. Previous observational results show a relationship between the wavelength of rolls and ABL depth, with shorter wavelengths associated with shallower mixed-layer depth (Weckwerth et al. 1997; Young et al. 2002). Analytical results indicate that the wavelength and depth of the HBL rolls are sensitive to the distribution of the mean wind shear profile and the inflection-point height (Foster 2005). The shorter wavelengths and larger residual velocities reported in WW98 are consistent with the high-shear 
environment of the eyewall region of Hurricane Fran where wind speeds approached $60 \mathrm{~m} \mathrm{~s}^{-1}$ in a very shallow inflow layer. As wind speeds increase near the eyewall of hurricanes, the depth of the surface layer can grow to $O(100 \mathrm{~m})$, whereas the depth of the mixed layer or HBL tends to decrease approaching the eyewall. Therefore, vortices originating in the surface layer may have increasing influence on the characteristics of the observed HBL rolls as one nears the radius of maximum winds and the eyewall.

In this study HBL roll signatures were generally absent in areas characterized by heavy convective rainbands outside the eyewall. It is suggested that within rainbands convectively unstable profiles dominate, with larger-scale overturning and downdrafts (Powell 1990a,b) that preclude inflection-point instability.

Wurman and Winslow find evidence of rolls in a region of heavy precipitation associated with the eyewall of Hurricane Fran. Static instability tends to be less in the eyewall than in surrounding spiral rainbands (Emanuel 1986), whereas shear and shear-induced turbulence are significantly greater in the eyewall HBL. Additionally, helical roll vortices that form just outside the eyewall may be advected into the eyewall region. Researchers have observed evidence of roll-like circulations embedded in the eyewall of some storms as seen and photographed from research aircraft flying inside the eye (P. Black 2004, personal communication). These considerations may help explain the presence of roll signatures in the heavy-rain region of the eyewall in Hurricane Fran. The radar data used in the research presented here were collected in regions outside the eyewalls of the hurricanes observed, with the exception of Georges' landfall in Puerto Rico (Fig. 5). Orographic influences associated with Puerto Rico's complex terrain may have disturbed roll structures during eyewall passage there, as clear roll signatures were not observed at that time.

Observational evidence presented here (Fig. 7) and theoretical results (Foster 2005) suggest that HBL rolls transport high-momentum air from the upper HBL downward, in agreement with past work (e.g., Lemone 1973; Glendening 1996; WW98). Over the ocean locally enhanced winds produced by the rolls result in anomalous dissipative heating, increased wave breaking, and an increased evaporation of sea spray (Businger and Businger 2001; Kepert 1996; Makin 1998). In combination these circumstances result in a locally enhanced latent heat flux and increased buoyancy of air, which is then entrained into the updraft region of the HBL roll. This overall process enhances the energy transport in the HBL and increases the efficiency of the oceanatmosphere energy flux. Over land, roll-enhanced wind gusts or streaks may be responsible for well-defined swaths of enhanced damage visible in forested areas following hurricane passage (Fujita 1992; Wakimoto and Black 1994).

Current HBL parameterizations using downgradient diffusion methods do not capture the nongradient mixing created by the HBL rolls. Estimates of the arealmean vertical momentum fluxes produced by the roll circulations (normalized by wavelength) are 2-3 times greater than the fluxes predicted by operational NWP model parameterizations of homogeneous turbulent diffusion. ${ }^{1}$ The mean height valid for the estimation of the roll-induced fluxes $(\sim 350 \mathrm{~m})$ is greater than that used in calculation of the flux due to homogeneous turbulent diffusion $(\sim 100 \mathrm{~m})$. It is suggested that the momentum flux will increase downward between 350and 100-m elevation. The reason is that the cross-isobar component of the flow toward lower pressure typically increases downward, due to the increasing impact of friction near the surface. Since cross-isobar flow represents a source of kinetic energy, in regions where the Lagrangian air parcel trajectories have a cross-isobar component, the momentum will increase downward. In this case, the roll-induced fluxes shown in Fig. 7 may underestimate the value valid at $100 \mathrm{~m}$.

The estimates for momentum fluxes presented in this paper were averaged over the rolls for comparison purposes. However, linear averaging is not appropriate when discussing nonlinear effects such as damage from wind gusts or dissipation of turbulence kinetic energy at the surface. The momentum flux associated with the velocity residuals, prior to calculation of an areal average, ranged from 7 to nearly $50 \mathrm{~m}^{2} \mathrm{~s}^{-2}$ and averaged $\sim 19 \mathrm{~m}^{2} \mathrm{~s}^{-2}$, significantly greater than the areal-average momentum flux shown in Fig. 7. These larger estimates may be appropriate to reference when considering the variability of the surface winds, their damage potential over land, and the impact of heating associated with the dissipation of turbulence kinetic energy at the air-sea surface (Businger and Businger 2001).

The drag coefficient over the ocean increases with wind speed for near-surface winds ranging from 5 to 30 $\mathrm{m} \mathrm{s}^{-1}$. For near-surface winds $\geq 40 \mathrm{~m} \mathrm{~s}^{-1}$ the drag coefficient becomes constant, or even decreases slightly (Powell et al. 2003). It has been suggested that the drag coefficient may approach its limit as the fraction of the

\footnotetext{
${ }^{1}$ Models referred to here include the National Centers for Environmental Prediction (NCEP) Global Forecast System, European Centre for Medium-Range Weather Forecasts (ECMWF) model, Geophysical Fluid Dynamics Laboratory (GFDL) Hurricane Model, and the Naval Research Laboratory (NRL) Nested Grid Operation Global Prediction System.
} 
ocean surface that is covered by breaking waves approaches $100 \%$. Downgradient diffusion holds that the magnitude of vertical fluxes depends on the magnitude of the drag coefficient; therefore compensating mechanisms must exist at high wind speeds to maintain the storm strength against increased dissipation. Persing and Montgomery (2003) suggest that heating in the eye may be important in this regard. HBL vortices may also play a key role in maintaining the high enthalpy fluxes necessary in the hurricane environment by transporting high enthalpy air upward into the HBL on the ascending side of the HBL rolls and lower enthalpy air downward on the descending side of the rolls. Assessment of the roll's impact on the ratio of the enthalpy and momentum exchange coefficients is a topic for future research.

It is the hope of the authors that the results presented in this paper, along with the questions and uncertainties raised, will promote interest in this area of research and provide motivation for further observational, theoretical, and modeling work in the HBL.

Acknowledgments. We thank Ralph Foster for helpful discussions and for input to the schematic shown in Fig. 8. We are grateful for the constructive comments of Josh Wurman and two anonymous reviewers. This research is supported by the National Science Foundation under Grant ATM-99-09011.

\section{REFERENCES}

Bakas, N. A., P. J. Ioannou, and G. E. Kefaliakos, 2001: The emergence of coherent structures in stratified shear flow. $J$. Atmos. Sci., 58, 2790-2806.

Bister, M., and K. A. Emanuel, 1998: Dissipative heating and hurricane intensity. Meteor. Atmos. Phys., 65, 233-240.

Brown, R. A., 1974: Analytical Methods in Planetary Boundarylayer Modeling. Wiley and Sons, 148 pp.

_ 1980: Longitudinal instabilities and secondary flows in the planetary boundary layer: A review. Rev. Geophys. Space Phys., 18, 683-697.

— , and L. Zeng, 2001: Comparison of planetary boundary layer model winds with dropsonde observations in tropical cyclones. J. Appl. Meteor., 40, 1718-1723.

Browning, K. A., and R. Wexler, 1968: The determination of kinematic properties of a wind field using Doppler radar. $J$. Appl. Meteor., 7, 105-113.

Businger, S., and J. A. Businger, 2001: Viscous dissipation of turbulent kinetic energy in storms. J. Atmos. Sci., 58, 3793-3796.

Carbone, R. E., M. J. Carpenter, and C. D. Burghart, 1985: Doppler radar sampling limitations in convective storms. J. Atmos. Oceanic Technol., 2, 357-361.

Christian, T. W., and R. M. Wakimoto, 1989: The relationship between radar reflectivities and clouds associated with horizontal roll convection on 8 August 1982. Mon. Wea. Rev., 117, $1530-1544$.
Crum, T. D., and R. L. Alberty, 1993: The WSR-88D and the WSR-88D operational support facility. Bull. Amer. Meteor. Soc., 74, 1669-1687.

Emanuel, K. A., 1986: An air-sea interaction theory for tropical cyclones. Part I: Steady-state maintenance. J. Atmos. Sci., 43, 585-604.

— 1999: Thermodynamic control of hurricane intensity. Nature, 401, 665-669.

Etling, D., and R. A. Brown, 1993: Roll vortices in the planetary boundary layer: A review. Bound.-Layer Meteor., 65, 215248.

Fleagle, R. G., and J. A. Businger, 1980: Atmospheric Physics. Academic Press, 432 pp.

Foster, R. C., 2005: Why rolls are prevalent in the hurricane boundary layer. J. Atmos. Sci., 62, 2647-2661.

Fujita, T. T., 1992: Damage survey of Hurricane Andrew in south Florida. Storm Data, Vol. 34, No. 8, 176 pp.

Glendening, J. W., 1996: Lineal eddy features under strong shear conditions. J. Atmos. Sci., 53, 3430-3449.

Katsaros, K. B., P. W. Vachon, P. G. Black, P. P. Dodge, and E. W. Uhlhorn, 2000: Wind fields from SAR: Could they improve our understanding of storm dynamics? John Hopkins APL Tech. Dig., 211, 86-93.

Kelly, R. D., 1982: A single Doppler radar study of horizontal-roll convection in a lake-effect snow storm. J. Atmos. Sci., 39, 1521-1531.

Kepert, J. D., 1996: Comments on "The temperature of evaporating sea spray droplets.” J. Atmos. Sci., 53, 1634-1645.

Kuettner, J., 1971: Cloud bands in the earth's atmosphere: Observations and theory. Tellus, 23, 404-425.

Lemone, M. A., 1973: The structure and dynamics of horizontal roll vortices in the planetary boundary layer. J. Atmos. Sci., 30, 1077-1091.

Lilly, D. K., 1966: On the instability of Ekman boundary flow. $J$. Atmos. Sci., 23, 481-490.

Makin, V. K., 1998: Air-sea exchange of heat in the presence of wind waves and spray. J. Geophys. Res., 103, 1137-1152.

Marks, F., P. Dodge, and C. Sandin, 1999: WSR-88D observations of hurricane atmospheric boundary layer structure at landfall. Preprints, $23 d$ Conf. on Hurricanes and Tropical Meteorology, Dallas, TX, Amer. Meteor. Soc., 1051-1054.

Mass, C. F., S. Businger, M. D. Albright, and Z. A. Tucker, 1995: A windstorm in the lee of a gap in a coastal mountain barrier. Mon. Wea. Rev., 123, 315-331.

Mourad, P. D., and B. A. Walter, 1996: Viewing a cold air outbreak using satellite-based synthetic aperture radar and Advanced Very High Resolution Radiometer imagery. J. Geophys. Res., 101, 16 391-16 400.

Persing, J., and M. T. Montgomery, 2003: Hurricane superintensity. J. Atmos. Sci., 60, 2349-2371.

Powell, M. D., 1990a: Boundary layer structure and dynamics in outer hurricane rainbands. Part I: Mesoscale rainfall and kinematic structure. Mon. Wea. Rev., 118, 891-917.

_ 1990b: Boundary layer structure and dynamics in outer hurricane rainbands. Part II: Downdraft modification and mixed layer recovery. Mon. Wea. Rev., 118, 918-938.

— P. J. Vickery, and T. A. Reinhold, 2003: Reduced drag coefficient for high wind speeds in tropical cyclones. Nature, 422, 279-283.

Stull, R. B., 1988: An Introduction to Boundary Layer Meteorology. Kluwer Academic, 206-208. 
Wakimoto, R. M., and P. G. Black, 1994: Damage survey of Hurricane Andrew and its relationship to the eyewall. Bull. Amer. Meteor. Soc., 75, 189-200.

Weckwerth, T. M., J. W. Wilson, R. M. Wakimoto, and N. A. Crook, 1997: Horizontal convective rolls: Determining the environmental conditions supporting their existence and characteristics. Mon. Wea. Rev., 125, 505-526.

Wroe, D. R., and G. M. Barnes, 2003: Inflow layer energetics of
Hurricane Bonnie (1998) near landfall. Mon. Wea. Rev., 131, $1600-1612$.

Wurman, J., and J. Winslow, 1998: Intense sub-kilometer boundary layer rolls in Hurricane Fran. Science, 280, 555-557.

Young, G. S., D. A. R. Kristovich, M. R. Hjelmfelt, and R. C. Foster, 2002: Rolls, streets, waves, and more: A review of quasi-two-dimensional structures in the atmospheric boundary layer. Bull. Amer. Meteor. Soc., 83, 997-1001. 